\title{
Ultra-low power Four Wave Mixing wavelength conversion in silicon micro-ring resonators with tunable Q-factor
}

\author{
M. J. Strain ${ }^{1,2}$, C. Lacava ${ }^{3}$, I. Cristiani ${ }^{3}$, M. Sorel ${ }^{2}$ \\ 1. Institute of Photonics, University of Strathclyde, Glasgow, G4 0NW, U.K \\ 2. School of Engineering, University of Glasgow, Glasgow, G12 8LT, U. K. \\ 3. Dipartimento di Ingegneria Industriale e dell'Informazione, Università di Pavia,Via Ferrata, 1, IT 27100 Pavia, Italy
}

The development of highly non-linear silicon devices is a fundamental step towards the realization of low power optical signal processing devices. In order to achieve this final goal several constraints have to be overcome. In particular, the next generation of opto-electronic integrated devices must be ultra-compact, compatible with foundry model fabrication processing and exhibit low power consumption [1]. In recent years, many non-linear photonic devices have been demonstrated based on the Silicon On Insulator (SOI) technology platform allowing for an unprecedented level of integration. Many research groups have demonstrated nonlinear devices that can perform wavelength conversion [2], demultiplexing operations [3] and all-optical switching [4]. In particular, in order to perform wavelength conversion, Four Wave Mixing (FWM) effects are typically used in silicon, showing conversion efficiencies that have ranged from $-40 \mathrm{~dB}[5]$ to $-12 \mathrm{~dB}$ with very high pump peak power, $>$. The main limitation of such silicon wavelength converter devices is related to the Two Photon Absorption (TPA) - excited Free Carriers (FC) that, at moderate pump power levels (>10 $\mathrm{mW}$ in a standard cross section silicon waveguide) introduces an additional loss that dramatically reduces the maximum achievable conversion efficiency [5]. Several techniques have been studied and demonstrated to reduce FC impact on FWM efficiency, including carrier removal techniques [5], silicon slot waveguides [ref], and amorphous silicon deposition instead of c-silicon [6]. However, these techniques suffer from several manufacturing constraints that make it extremely challenging to fabricate them within a foundry model paradigm. In order to decrease the pump power, and consequently reduce the impact of generated FC's, resonant devices has been considered and demonstrated in the past few years [7]. In such structures the nonlinear response is drastically improved due to the field enhancement of the cavity, which can be evaluated as a function of the Q-factor. The latter is defined by the roundtrip loss and coupling coefficient and is typically a static property of the device. In this work control is exerted over both the device Q-factor and the resonant wavelengths using simple electrical contacts, allowing direct modulation of resonance enhanced processes such as Four Wave Mixing (FWM).

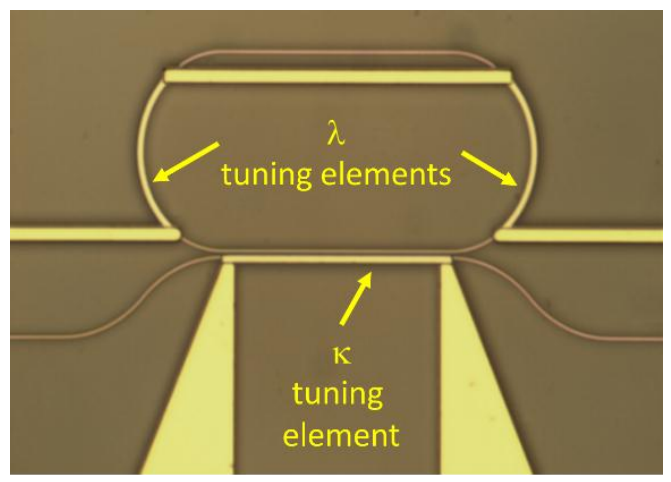

Fig. 1: Optical micrograph of a micro-ring resonator with tunable coupler and ring sections.

Compact silicon microring resonators have been demonstrated with Q-factors in the $10^{5}$ range [8]. However, the critical coupling condition in such high $\mathrm{Q}$ resonators exhibits a extreme sensitivity to fabrication tolerances in manufacture, causing variation in the resonator optical path length, losses and coupling coefficient. Nanometre scale fabrication variations can significantly detune the resonator from its designed condition and hence degrade the resonant enhancement of the non-linear processes. 


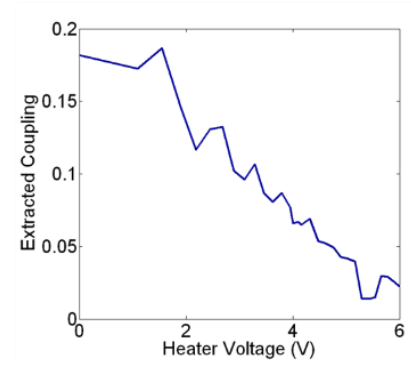

(a)

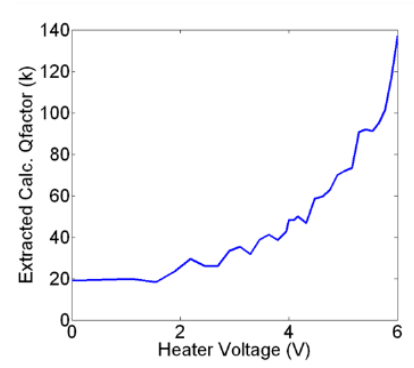

(b)

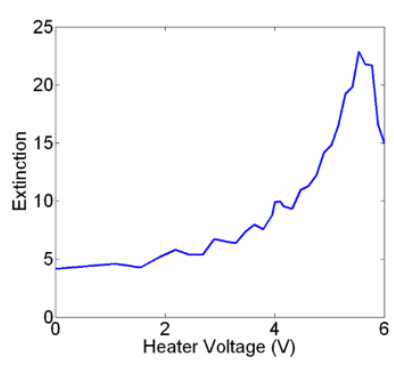

(c)

Fig. 2: (a) Coupling coefficient, (b) Q-Factor and (c) Extinction as a function of the voltage applied on the coupling heater

In this work, by electrically controlling the power coupling coefficient to the ring, in addition to its resonant wavelength, the device Q-factor can be tuned. The device geometry is shown in Fig.1. The critical coupling point of the resonator can be easily attained so long as the device is designed to be over-coupled. Any fabrication variation causing a shift in coupling coefficient can therefore be overcome. Electrical control is excercised using a resistive heating element placed assymetrically over the evancescent field coupler [9]. The heater element generates a thermal gradient across the two waveguides of the evanescent field coupler and hence detunes their effective refractive indices, varying the power coupled to the device, and therefore its Qfactor (see Fig.2). Resonators, designed with a coupling coefficient of 0.2 exhibited tuning down to 0.02, corresponding to a Q-factor variation between $20 \times 10^{3}$ and $140 \times 10^{3}$ as shown in Fig.2(b). By tuning an optimised coupled ring to its critical coupling point, a resonant Q-factor of $100 \times 10^{3}$ was achieved. FWM experiments, Fig. 3, were carried out and a conversion efficiency of $-10.9 \mathrm{~dB}$ was measured with an input power on-chip of only $0.78 \mathrm{~mW}$. Finally, FWM efficiency was measured as a function of the ring-coupling coefficient, showing that an ehnancement of $30 \mathrm{~dB}$ is achieved when the critical coupling point is reached (Fig. 3(b)), with high sensitivity of the efficiency to the coupling coefficient around this point.

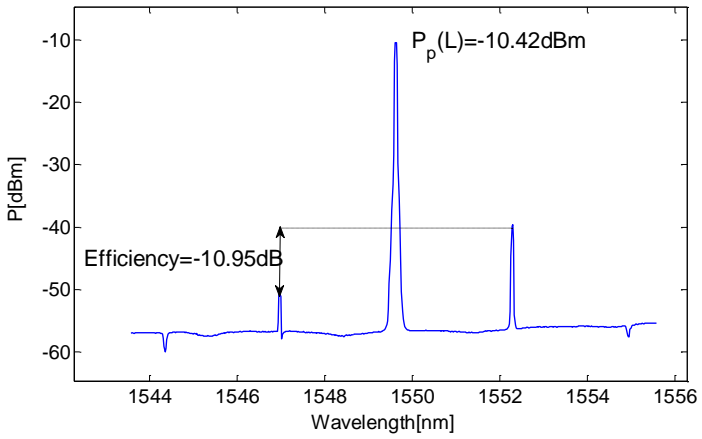

(a)

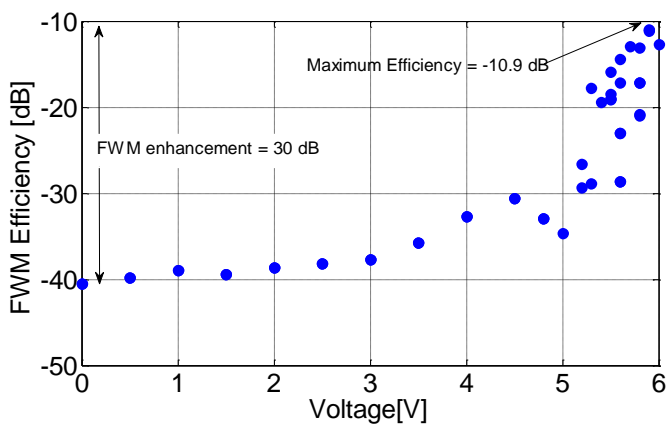

(b)

Fig. 3: (a) FWM measured spectra at the critical coupling point; (b) FWM efficiency as a function of the applied voltage

\section{References:}

[1] Almeida, V., Barrios, C., Panepucci, R., \& Lipson, M. (2004). All-optical control of light on a silicon chip. Nature, 431(October), $1081-1084$. doi:10.1038/nature02925.1

[2] Foster, M., Turner, A. C., Salem, R., Lipson, M., \& Gaeta, A. L. (2007). parametric wavelength conversion in silicon nanowaveguides. Optics Express, 15(20), 12949-12958.

[3] Driscoll, J., Astar, W., \& Liu, X. (2010). All-optical wavelength conversion of $10 \mathrm{~Gb} / \mathrm{s}$ RZ-OOK data in a silicon nanowire via cross-phase modulation: Experiment and theoretical investigation. Selected Topics in Quantum Electronics, IEEE Journal of, 16(5), 1448-1459.

[4] Lacava, C., Strain, M. J., Minzioni, P., Cristiani, I., \& Sorel, M. (2013). Integrated nonlinear Mach Zehnder for 40 Gbit/s all-optical switching. Optics Express, 21(18), 21587

[5] Rong, H., Kuo, Y.-H. Y., Liu, A., Paniccia, M., \& Cohen, O. (2006). High efficiency wavelength conversion of $10 \mathrm{~Gb} / \mathrm{s}$ data in silicon waveguides. Optics Express, 14(3), 1182-1188.

[6] Lacava, C., Minzioni, P., Baldini, E., Tartara, L., Fedeli, J. M., \& Cristiani, I. (2013). Nonlinear characterization of hydrogenated amorphous silicon waveguides and analysis of carrier dynamics. Applied Physics Letters, 103(141103).

[7] Morichetti, F., Canciamilla, A., Ferrari, C., Samarelli, A., Sorel, M., \& Melloni, A. (2011). Travelling-wave resonant four-wave mixing breaks the limits of cavity-enhanced all-optical wavelength conversion. Nature Communications, 2(May), 1-8.

[8] P. De Heyn, D. Vermeulen, T. Van Vaerenbergh, B. Kuyken, and D. Van Thourhout, "Ultra-high Q and finesse all-pass microring resonators on Silicon-on-Insulator using rib waveguides,” In Proc. 16th European Conference on Integrated Optics, (2012) 
[9] P. Orlandi, F. Morichetti, M. J. Strain, M. Sorel, A. Melloni, and P. Bassi, "Tunable silicon photonics directional coupler driven by a transverse temperature gradient.," Opt. Lett., vol. 38, no. 6, pp. 863-5, Mar. 2013. 